\title{
Canons of Collaborative Care
}

\author{
Kurt Kroenke, $M D^{7}$ (i) and Andrea Cheville, $M D^{2}$
}

'Division of General Internal Medicine and Geriatrics, Indiana University School of Medicine and Regenstrief Institute, Inc., Indianapolis, IN, USA;

${ }^{2}$ Department of Physical Medicine and Rehabilitation, Mayo Clinic, Rochester, MN, USA.

J Gen Intern Med 37(2):456-8

DOI: $10.1007 / \mathrm{s} 11606-021-06929-9$

() Society of General Internal Medicine 2021

$\mathrm{C}$ ollaborative care (CC) is a team-based model in which the patient's primary clinician (PC) is assisted in the management of specific health conditions by a care manager (CM) who is typically supervised by a physician with special expertise in the management of those conditions. ${ }^{1,2} \mathrm{CC}$ was initially developed in the 1980s to detect and manage depression in primary care. However, past decades have witnessed broad CC generalization to other contexts, populations, and clinical targets. There have been more than 80 randomized trials of CC targeting depression, pain, and anxiety. Recent efforts have further extended CC to address co-occurring symptoms and functional decline. Although variants of CC have also been tested for common medical conditions, the latter tend to have stronger evidence-based guidelines, greater physician training and competency, and clearer specialty referral pathways. Thus, a major niche for $\mathrm{CC}$ is targeted care for symptoms and functional decline, areas too frequently marginalized in medically oriented care delivery systems. The potential positive impact of CC expansion cannot be overstated considering an aging population as well as an increased prevalence of multi-morbidity, mental disorders, chronic pain, and other symptom-based conditions.

Earlier CC studies predominantly used a nurse CM collaborating with primary care clinicians to improve management of a single target condition. In newer CC iterations, the CM role has been filled by clinicians other than a nurse (e.g., pharmacist, social worker, physical therapist); the $\mathrm{CC}$ team has partnered with diverse specialists (e.g., oncology, neurology, cardiology); and targets have increased to as many as six conditions. $^{3-5}$ In all CC iterations, the team's principal tasks include patient education and enhanced self-management; monitoring outcomes, adherence, and side effects; and adjusting treatment. Useful CM skills may extend well beyond medication management, to include motivational interviewing and behavioral activation. Integrated care and CC are used

Received April 17, 2021

Accepted May 11, 2021

Published online June 2, 2021 interchangeably. Efforts to extend CC have highlighted the tension between adapting the approach to accommodate contextual and target requirements while preserving defining $\mathrm{CC}$ characteristics with fidelity.

One definition of canon is a principle, criterion, standard, or norm within a particular field of study or discipline. Four canons with subsidiary principles are summarized in Table $\mathbf{1 .}$ Although core components of $\mathrm{CC}$ have been elaborated elsewhere $e^{1,2,6}$, the canons proposed here emphasize some pragmatic aspects of operationalizing $\mathrm{CC}$ derived from our experience in testing $\mathrm{CC}$ in multiple trials and implementing it in clinical practice.

\section{OWN THE TARGET CONDITION}

The raison d'étre of CC is to provide resources for conditions difficult to adequately manage within the time constraints that beset the patient's PC. Thus, CC that creates extra work for the $\mathrm{PC}$ can be a self-defeating nonstarter. An illustrative formula would be the difference between work added and work subtracted by $\mathrm{CC}$, with 3 scenarios:

1. Work $_{\text {added }}-$ Work $_{\text {subtracted }}=1-2$. This is a net PC work reduction provided by $\mathrm{CC}$

2. Work $_{\text {added }}-$ Work $_{\text {subtracted }}=2-2$. This is a net PC work "zero sum game"

3. Work $_{\text {added }}-$ Work $_{\text {subtracted }}=2-1$. This is a net PC work added by $\mathrm{CC}$

Scenario 3 should be the exception rather than the norm. The CC team also needs to be very selective about PC communications given the daily volume of secure messages and other alerts overloading the clinician's inbox. PCs are as much "clients" of the CC team as the patients, and their needs must be respected.

\section{ELICIT PATIENT PREFERENCES}

Patient preferences may be relevant at several levels:

1. Does the patient want any treatment? Symptoms identified by screening may not be sufficiently bothersome or their management a lower priority for the patient compared to other medical or non-medical issues.

2. If multiple evidence-based treatments are available, which does the patient prefer? 
Table 1 Canons of Collaborative Care

\section{Own the target condition.}

a CC does the "heavy lifting" for the condition being targeted and mainly involves the primary clinician (PC) for a couple reasons. - The PC needs to order a test, treatment, or specialty referral

- There are "red flags" indicating something serious or urgent

b Don't create work for the PC. Subtract work.

c Communicate with the PC occasionally rather than often. Limit messaging to $\mathrm{PC}$ actions that are required rather than simply FYI (i.e., for your information). Also, inform the $\mathrm{PC}$ when new treatments are initiated or changed as well as if the patient declines treatment.

$2 \quad$ Elicit patient preferences

a Does the patient want any treatment for the condition? If not, explore why and determine whether motivation or education is important at this point, vs. watchful waiting

b Prioritize which symptom the patient desires treatment for, if more than one symptom

c Offer a menu of treatment options if there are multiple evidencebased treatments.

d Treatment algorithms are guidelines and not commandments

e Manage expectations.

- Gradual and incremental change (go for a first down rather than a touchdown)

- Symptom improvement rather than eradication is the modal outcome

$3 \quad$ Measure and adjust

a Explain rationale for measurement. Measure symptom at periodic intervals., including:

- Symptom severity using validated patient-reported outcome (PRO) measure

- Global status (impairment at baseline; change [same, better,

worse] at follow-up)

- Patient desire for treatment initiation or change

b Assess and diagnose non-adherence. Common reasons include cost or accessibility of treatment; side effects; ineffectiveness of treatment; complexity of regimen.

c Find the "sweet spot" between measurement-based care and excessive measurement. Don't measure domains you are not going to act upon

d Adjust treatments if not working (increase dose; change treatment; combine treatments)

4 Determine the $\mathrm{CC}$ team's boundaries of care

a Don't get diverted by non-target (out of scope) conditions - Understand the problem (empathize) but don’t undertake its management

- Don't dismiss or minimize the problem. However, say more than "Talk to your doctor about it." Consider next steps the patient might take without $\mathrm{CC}$ assuming care.

b Determine "discharge" policies (i.e., when to transition all care back from $\mathrm{CC}$ to $\mathrm{PC}$ )

- Sign off if improvement is sufficient or treatment is no longer needed or desired

- Determine referral procedures for symptoms refractory to $\mathrm{CC}$ or which require specialist evaluation, treatments, or procedures

- Consider how long CC should follow a patient. Is there a limit on number of weeks or number of contacts before

"reauthorization" is required?

c Explicitly inform the patient of the boundaries of CC care and its time-limited nature

3. If the patient has multiple symptoms that fall within the purview of the CC team (e.g., pain, depression, and trouble sleeping), which symptom is most salient for the patient? Sequential rather than simultaneous treatment may be preferable. Moreover, treatment of the most bothersome symptom may have beneficial effects on comorbid symptoms. It may be better to focus on one problem well than three problems halfway.
4. If there is more than one recommendation from the same or different consultants within or outside the CC team, each potential action is probably partly right without any being necessarily superior to the others. That gives a menu to choose from rather than an overly prescriptive course of action. In this case, the care manager can navigate with the patient an initial option with the possibility of a course correction should symptom improvement be sub-optimal.

\section{MEASURE AND ADJUST}

A core principle of $\mathrm{CC}$ is measurement-based care in which validated scales are periodically administered to monitor and adjust therapy. Treat-to-target is a corollary principle wherein the focus is on the measured outcome rather than treatment specifics. While scale scores have been a principal focus, adherence, side effects, and perceived cost are measurable parameters that should also guide adjustments. If the patient is adherent to a partially effective treatment, one option is to increase the dose (if a medication) or number of sessions (if a nonpharmacological therapy). Another option is to add an additional treatment with a different mechanism of action. If a treatment is not effective or the patient desires an alternative treatment, the team can return to the original menu of evidence-based options.

One caveat for measurement-based care is to avoid treating a scale score in isolation. Two other factors noted in Table 1 are global status and the patient's desire for treatment. At baseline, global status refers to a symptom's impact on a patient's overall well-being. At follow-up, global status refers to the degree of change in this broader construct. Symptom's temporal characteristics and etiology may also inform treatment. A patient might tolerate a pain score of 8 for a few days but not a pain score of 5 for six months. Patient-specific thresholds for symptom tolerance (i.e., the degree of symptom hypersensitivity vs. stoicism) may also differentially guide symptom management. An obvious and transient symptom source may indicate the need for a time-limited, near-term solution rather than a durable one. Finally, lower symptom scores may warrant expedited action in the presence of red flags, such as suicidal ideation for depression or back pain in a patient with cancer adjacent to the spinal cord.

\section{DETERMINE THE CC TEAM'S BOUNDARIES OF CARE}

$\mathrm{CC}$ is frequently bounded by the conditions it covers, their cause, and length of time the patient is followed. Dealing with conditions outside its scope and transitioning care back to PC is addressed in Table 1. When CC is provided to patients in specialty settings, even the condition covered may be better managed elsewhere. For example, $\mathrm{CC}$ targeting cancer-related 
pain in oncology practices or postoperative pain in surgical settings may not be the ideal service for addressing preexisting back pain, fibromyalgia, or chronic headache. In these cases, re-directing the patient to the appropriate primary care or specialty service is appropriate. Explicitly communicating to the patient the boundaries of CC and its time-limited nature is important.

Relative to the evidence for $\mathrm{CC}$, its adoption has been largely limited to selected health care systems with a more population-based approach. However, uptake could be enhanced by recent reimbursement changes ${ }^{7}$, accountable health care initiatives, and the increased use of telecare accelerated by but likely to persist after the pandemic. The canons codify some practical strategies for a more seamless integration of CC.

Corresponding Author: Kurt Kroenke, MD; Division of General Internal Medicine and Geriatrics, Indiana University School of Medicine and Regenstrief Institute, Inc., Indianapolis, IN, USA (e-mail: kkroenke@regenstrief.org).

\section{Declarations:}

Conflict of Interest: Neither of the authors have a conflict of interest.

\section{REFERENCES}

1. Huffman JC, Niazi SK, Rundell JR, Sharpe M, Katon WJ. Essential articles on collaborative care models for the treatment of psychiatric disorders in medical settings: a publication by the academy of psychosomatic medicine research and evidence-based practice committee. Psychosomatics. 2014;55(2): 109-122.

2. Kroenke $\mathbf{K}$, Unutzer $\mathbf{J}$. Closing the false divide: sustainable approaches to integrating mental health services into primary care. J Gen Intern Med 2017;32:404-10

3. Cheville AL, Moynihan T, Herrin J, Loprinzi C, Kroenke K. Effect of collaborative telerehabilitation on functional impairment and pain among patients with advanced-stage cancer: a randomized clinical trial. JAMA Oncology 2019;5(5):644-653.

4. Rutten LJ, Ruddy KJ, Chlan LL, Griffin JM, Herrin J, Leppin AL, Pachman DR, Ridgeway JL, Rahman PA, Storlie CB, Wilson PM, Cheville AL. Pragmatic cluster randomized trial to evaluate effectiveness and implementation of enhanced EHR-facilitated cancer symptom control (E2C2). Trials. 2020;21(1):1-19.

5. Kroenke K, Baye F, Lourens SG, Evans E, Weitlauf S, McCalley S, Porter B, Matthias MS, Bair MJ. Automated self-management (ASM) vs. ASM-enhanced collaborative care for chronic pain and mood symptoms: the CAMMPS randomized clinical trial. J Gen Intern Med 2019;34(9): 1806-1814

6. Overbeck, G., Davidsen, A.S. \& Kousgaard, M.B. Enablers and barriers to implementing collaborative care for anxiety and depression: a systematic qualitative review. Implementation Sci 2016;11:165. https://doi.org/10. 1186/s13012-016-0519-y

7. Carlo AD, Unützer J, Ratzliff AD, Cerimele JM. Financing for collaborative care-a narrative review. Current treatment options in psychiatry. 2018;5(3):334-44

Publisher's Note: Springer Nature remains neutral with regard to jurisdictional claims in published maps and institutional affiliations. 